\title{
Application of Self-Adaptive Wavelet Ridge Demodulation Method Based on LCD to Incipient Fault Diagnosis
}

\author{
Songrong Luo, ${ }^{1,2}$ Junsheng Cheng, ${ }^{2}$ and Jianping $\mathrm{Fu}^{2}$ \\ ${ }^{1}$ College of Mechanical Engineering, Hunan University of Arts and Science, Changde 415003, China \\ ${ }^{2}$ China College of Mechanical and Vehicle Engineering, Hunan University, Changsha 410082, China \\ Correspondence should be addressed to Songrong Luo; luosr7351@sina.com
}

Received 19 November 2014; Revised 20 March 2015; Accepted 5 April 2015

Academic Editor: Lei Zuo

Copyright (c) 2015 Songrong Luo et al. This is an open access article distributed under the Creative Commons Attribution License, which permits unrestricted use, distribution, and reproduction in any medium, provided the original work is properly cited.

\begin{abstract}
When a local defect occurs in gearbox, the vibration signals present as the form of multicomponent amplitude modulation and frequency modulation (AM-FM). Demodulation analysis is an effective way for this kind of signal. A self-adaptive wavelet ridge demodulation method based on LCD is proposed in this paper. Firstly, multicomponent AM-FM signal is decomposed into series of intrinsic scale components (ISCs) and the special intrinsic scale component is selected in order to decrease the lower frequency background noise. Secondly, the genetic algorithm is employed to optimize wavelet parameters according to the inherent characteristics of signal; thirdly, self-adaptive wavelet ridge demodulation wavelet for the selected ISC component is performed to get instantaneous amplitude (IA) or instantaneous frequency (IF). Lastly, the characteristics frequency can be obtained to identify the working state or failure information from its spectrum. By two simulation signals, the proposed method was compared with various existing demodulation methods; the simulation results show that it has higher accuracy and higher noise tolerant performance than others. Furthermore, the proposed method was applied to incipient fault diagnosis for gearbox and the results show that it is simple and effective.
\end{abstract}

\section{Introduction}

Fault diagnosis technique is of great significance to guarantee the normal operation of mechanical and electrical equipment. When a localized defect occurs in gearbox, the vibration signals present as the form of multicomponent amplitude modulation and frequency modulation (AM-FM) [1], expressed as a frequency family on the spectrum, which contains the center frequency and its harmonic frequency. For this kind of signals, some demodulation techniques have been used to find the fault feature information. Hilbert demodulation method is widely used in machinery fault diagnosis $[2,3]$, but there exists window effect and end effect of Hilbert transforms inevitably, resulting in greater demodulation error. The energy separation algorithm (ESA) appears much popular in recent years for the application to machinery fault diagnosis [4-7], because it is suitable to extract the local dynamic characteristics of nonstationary signal. However, ESA requires that the processed signal should be narrow-band monocomponent $[4,5]$. In addition, ESA is sensitive to noise [8]. Compared with the above time domain demodulation methods, the wavelet ridge demodulation technique is time-frequency domain demodulation method, which uses continuous wavelet transform (CWT) to obtain instantaneous amplitude (IA) information and instantaneous frequency (IF) information $[8,9]$. In general, the analytic Morlet wavelet is used as the basic wavelet due to its similarity to the fault associated impacts [1013]. But, the analytic Morlet wavelet parameters, which are center frequency and shape factor, would exert a great impact on the results of wavelet ridge demodulation. In order to select the proper parameters, some techniques have been employed [10-12]. Unfortunately, there is no mature theory to tell us how to choose them. In addition, there are few methods, which can select both center frequency and shape factor of Morlet wavelet to obtain the optimal timescale resolution. Here, genetic algorithm (GA), which not only has better ability to search the optimal solution but 
also has fast convergence, is introduced to obtain the two optimal parameters according to the analyzed signal local characteristics, and Morlet wavelet with optimal parameters using GA is called self-adaptive wavelet. Therefore we will utilize self-adaptive wavelet ridge demodulation approach to obtain better demodulation results in this paper.

On the other hand, to greatly eliminate the background noise and improve the demodulation accuracy, multicomponent AM-FM signals should be decomposed into monocomponent AM-FM signals before using self-adaptive wavelet ridge demodulation approach. Empirical mode decomposition (EMD) method $[3,4,14,15]$ or local mean decomposition (LMD) method [16-18] is widely employed to decompose multicomponent AM-FM signal into monocomponent AMFM signals in general. However, EMD method still has theoretical limitations, such as frequency confusion, overshooting, undershooting, end effect, and the emergence of negative frequency components of nonphysical meaning. Compared to the EMD method, LMD method avoids these problems to some extent, but its computing speed is much slower than EMD. Local characteristic-scale decomposition (LCD) is a new data-driving signal analysis method. Based on the inherent characteristics of the signal itself, the LCD method can decompose a complex multicomponent AMFM into several intrinsic scale components (ISC). Simultaneously, each ISC component is a monocomponent AMFM signal which has obvious physical meaning. Our team's research works show that compared with the LMD and EMD method, LCD not only avoids the shortcomings of EMD and LMD, but also owns much faster computing speed [19-21]. Therefore, LCD method is used to decompose the multicomponent gearbox fault vibration signal to a number of ISCs at first. Subsequently, one or several interesting ISCs are selected as analyzed component. After that, noise would be greatly removed to clearly demodulate fault-associated features component from the selected ISCs.

In summary, targeting the demodulation solution of the multicomponent AM-FM vibration signal with low signalnoise ratio produced by gearbox failures, we present a selfadaptive wavelet ridge demodulation method based on LCD for fault diagnosis. The rest of the paper is organized as follows. In Section 2, the wavelet ridge demodulation principle based on LCD is introduced. The process to get a self-adaptive wavelet based on GA is described. We describe the proposed method and the simulation study is provided in Section 4. The proposed method is applied for incipient fault diagnosis of gearbox in Section 5. Finally, we offer conclusions in Section 6.

\section{Wavelet Ridge Demodulation Principle Based on LCD}

A real signal of monocomponent can be expressed as $s(t)=A(t) \cos (\varphi(t))$. When the instantaneous frequency of the signal is much larger than the amplitude modulation frequency, expressed as $|d \varphi(t) / d t| \gg|(1 / A(t))(d A(t) / d t)|$, this real signal monocomponent is called a progressive single frequency signal. Then its analytical signal can be written as

$$
Z_{s}(t)=A_{s}(t) \exp \left(j \varphi_{s}(t)\right)
$$

The expression of the instantaneous frequency is

$$
f_{s}=\frac{1}{2 \pi} \frac{d \varphi_{s}(t)}{d t}
$$

After selecting a mother wavelet $\psi(t)$ whose analytical wavelet is expressed as $\tilde{\psi}(t)=A_{\psi}(t) \exp \left(j \varphi_{\psi}(t)\right), Z_{s}(t)$ can be transformed by continuous wavelet transformation as follows:

$$
W_{z}(a, b)=\frac{1}{\sqrt{a}} \int_{-\infty}^{\infty} A_{a, b}(t) \exp \left(j \varphi_{a, b}(t)\right) d t,
$$

where $a$ is the scale parameter, $b$ is the translation parameter:

$$
\begin{aligned}
& A_{a b}(t)=A_{s}(t) A_{\psi}\left(\frac{t-b}{a}\right), \\
& \varphi_{a b}(t)=\varphi_{s}(t)-\varphi_{\psi}\left(\frac{t-b}{a}\right) .
\end{aligned}
$$

As to any scale parameter $a$ and translation parameter $b$, suppose $\varphi_{a b}(t)$ only has a first-order stagnation $t_{s}=t_{s}(a, b)$, and then the first-order stagnation satisfies $\varphi_{a b}^{\prime}\left(t_{s}\right)=0$ and $\varphi_{a b}^{\prime \prime}\left(t_{s}\right) \neq 0$; that is,

$$
\varphi_{s}^{\prime}\left(t_{s}\right)=\frac{1}{a} \varphi^{\prime}\left(\frac{t_{s}-b}{a}\right) .
$$

Wavelet ridge is defined as a collection of all the points which meet $t_{s}(a, b)=b$ on a phase plane. The expression of the collection is $R=\left\{(a, b) \in H^{2}(R), t_{s}(a, b)=b\right\}$, where $H^{2}(R)$ is a Hardy real space. A point $\left(a_{r}(b), b\right)$ on the wavelet ridge line is called wavelet ridge point. Obviously, according to formula (5), there is

$$
a=a_{r}(b)=\frac{\varphi_{\psi}^{\prime}(0)}{\varphi_{s}^{\prime}(b)} .
$$

Here can be seen that instantaneous frequency can be extracted from the wavelet ridge points.

Also, the wavelet coefficients of signal $s(t)$ about $\tilde{\psi}(t)$ can be expressed as

$$
\begin{aligned}
W_{s}(a, b) \approx & \frac{\sqrt{a}}{2} A_{s}(b) \exp (j \varphi(b)) \\
& \times\left(\widehat{\psi}\left(a\left[\frac{\omega_{0}}{a}-\varphi_{s}^{\prime}(b)\right]\right)\right),
\end{aligned}
$$

where $\widehat{\psi}(\omega)$ is the Fourier transform of $\psi(t)$. And the wavelet coefficients modulus is initially defined as

$$
\left|W_{s}(a, b)\right| \approx \frac{\sqrt{a}}{2} A_{s}(b)\left|\widehat{\psi}\left(a\left[\frac{\omega_{0}}{a}-\varphi_{s}^{\prime}(b)\right]\right)\right| .
$$


As to wavelet ridge point, it can be seen that $\omega_{0} / a_{r}-$ $\varphi_{s}^{\prime}(b)=0$ from formula (6). So the wavelet coefficients modulus of wavelet ridge point is further expressed as

$$
\left|W_{s}\left(a_{r}, b\right)\right| \approx \frac{\sqrt{a}}{2} A_{s}(b)|\widehat{\psi}(0)| .
$$

So, it can be seen that signal instantaneous frequency can be gained after the wavelet ridge is extracted, which is expressed as

$$
f_{s}(t)=\frac{1}{2 \pi} \frac{\omega_{0}}{a_{r}(t)}
$$

where $\omega_{0}$ is the center frequency of $\tilde{\psi}(t)$; that is, $\omega_{0}=\varphi_{\psi}^{\prime}(0)$.

At the same time, the signal instantaneous amplitude can be expressed as

$$
A_{s}(t) \approx \frac{2\left|W_{s}\left(a_{r}(t), t\right)\right|}{\sqrt{a_{r}(t)}|\widehat{\psi}(0)|} .
$$

As presented above, it is clear that the demodulation analysis of monocomponent AM-FM signal based on wavelet ridge is feasible. However, most vibration signals produced by gearbox failures are generally multicomponent AM-FM signals. They should be decomposed into monocomponent AMFM signals by appropriate time-frequency signal processing method before demodulation. In this paper, LCD method is employed to accomplish the signal decomposition.

The LCD method has the assumptions that a complex signal consists of a number of ISCs (Intrinsic Scale Component, ISC) and any two ISCs are independent of each other. In the entire data segment, ISC must meet the following two conditions.

(I) The maximal value is positive, the minimum value is negative, and the data set are monotonic between any two adjacent extreme points.

(II) Let all the extreme points be written as $\left(X_{k}, \tau_{k}\right)$, $k=1,2, \ldots, M$; the line $l_{k}$ determined by any two adjacent extreme points $\left(X_{k}, \tau_{k}\right)$ and $\left(X_{k+2}, \tau_{k+2}\right)$ can be expressed as

$$
l_{k}=\frac{X_{k+2}-X_{k}}{\tau_{k+2}-\tau_{k}}\left(t-\tau_{k}\right)+X_{k} .
$$

Remembering the value of $l_{k}$ at the $\tau_{k+1}$ as $A_{k+1}$, the relation between $A_{k+1}$ and $X_{k+1}$ should meet the following:

$$
\alpha A_{k+1}+(1-\alpha) X_{k+1}=0
$$

when $\alpha=0.5, A_{k+1}=-X_{k+1}$.

Based on this definition, a complex multicomponent AMFM signal can be decomposed into the sum of a finite number of ISCs and a residual signal. Each ISC is a monocomponent AM-FM signal whose instantaneous frequency has specific physical meaning. That is,

$$
x(t)=\sum_{i=1}^{N} c_{i}(t)+u(t),
$$

where $c_{i}(t)$ is the $i$ th ISC component and $u(t)$ is the residual signal.

\section{Self-Adaptive Wavelet}

3.1. Morlet Wavelet Frequency Resolution. When there is local failure for gear, the fault gear teeth will stimulate system to produce a convergent impact response and the vibration signal collected by the acceleration sensor shows the obvious multicomponent modulation characteristic. Therefore, as mentioned above, we can adopt wavelet ridge demodulation based on LCD to extract the fault feature. In order to match this kind of signal, analytic Morlet wavelet with impact feature is chosen, which is defined as

$$
\tilde{\psi}(t)=\frac{1}{\sqrt{f_{b} \pi}} \exp \left(-\frac{t^{2}}{f_{b}}\right) \exp \left(j 2 \pi f_{c} t\right) .
$$

The Fourier transform of $\psi(t)$ is represented as

$$
\widehat{\psi}(2 \pi f)=\exp \left[\left(-\frac{2 \pi f-2 \pi f_{c}}{2 \sqrt{f_{b}}}\right)^{2}\right],
$$

where $f_{b}$ is the shape factor and $f_{c}$ is the center frequency, whose numerical values determine the speed of the waveform vibration damping, respectively; from formula (16), Morlet wavelet quality factor is $Q=\sqrt{2} \pi f_{c} \sqrt{f_{b}} / \sqrt{\ln 2}$. So, the best frequency resolution can be gained by adjusting $f_{b}$ and $f_{c}$, which can result in a good time-scale accumulation.

3.2. The Procedure of Obtaining Self-Adaptive Wavelet. Sparse degree of wavelet coefficients can characterize the degree of similarity between the basic wavelet function and signal. The energy entropy of wavelet can indicate this sparse degree, which shows accumulation performance of wavelet coefficients. As to each specific scale $j$, the wavelet energy entropy is defined as

$$
W_{E}=-\sum_{j} P_{j} \ln P_{j}
$$

where $P_{j}=E_{j} / E_{T}$ is the probability of energy distribution $\left(\sum P_{j}=1\right), E_{j}=\int W^{2}(j, \tau) d \tau$ is the wavelet energy, $W(j, \tau)$ is the wavelet coefficient, and $E_{T}=\sum_{j} E_{j}$ is the total wavelet energy within the time scale plane.

Accordingly, the wavelet energy entropy is taken as the objective function during selecting the optimal wavelet parameters. To optimize wavelet parameters, genetic algorithm (GA) and particle swarm optimization (PSO) [22, 23] are two widely utilized approaches. In this paper, GA is employed to optimize either envelope factor or center frequency with wavelet energy entropy as the fitness function. That wavelet with optimal parameters is called selfadaptive wavelet. The procedure to get self-adaptive wavelet is described as follows.

Step 1. Set search prime range and population size of parameters, $f_{b}$ and $f_{c}$, and randomly generate initial population. In this paper, the population size is set to 100 and the parameters are, respectively, encoded as 10-bit binary string chromosomes by binary coding method. 
Step 2. Make wavelet decomposition of the signal and calculate the fitness value of each individual according to formula (17). Then, sort the fitness values by size.

Step 3. Based on individual fitness value in the search space, individuals are screened and evolved by a series of genetic manipulation, selection, reproduction, crossover, mutation, and so forth to constantly update and select populations.

Step 4. In this step, it is determined whether iteration satisfies the termination condition or not. If satisfied, the optimal solution is finished. If not satisfied, go to Step 2 until the optimal solution is got. The optimization procedure of wavelet parameters via GA is shown in Figure 1.

\section{The Proposed Method and Simulation}

Since demodulation technique is an effective way to reveal the fault characteristic frequency for fault diagnosis of gearbox, a self-adaptive wavelet ridge demodulation based on LCD for fault diagnosis is proposed in this paper. Firstly, LCD method is adopted to decompose the original signal into a number of ISC components, and lower frequency noise is decreased by selecting special ISC component which contains rich fault feature. Secondly, the genetic algorithm is used to optimize wavelet parameters to obtain self-adaptive wavelet based on wavelet energy entropy which is served as the objective function. Thirdly, the self-adaptive wavelet ridge demodulation for the selected ISC component is used to extract dynamic information. Finally, the characteristics frequency can be obtained to identify the working state or failure information from the spectrum. The flowchart of the proposed fault diagnosis method was illustrated in Figure 2.

To verify the validity of the proposed method, let us consider the following signal:

$$
\begin{aligned}
x(t) & =x_{1}(t)+x_{2}(t), \\
x_{1}(t) & =(1+0.5 \cos 20 \pi t) \sin (200 \pi t+2 \cos 20 \pi t), \\
x_{2}(t) & =\sin \pi t \sin 20 \pi t \quad t \in[0,1] .
\end{aligned}
$$

Obviously, $x(t)$ is a complex multicomponent AM-FM signal, containing AM-FM component $x_{1}(t)$ and AM component $x_{2}(t)$. The sampling frequency is $1000 \mathrm{~Hz}$. Time domain waveforms of simulation signal $x(t)$ and its LCD decomposition results are shown in Figure 3, where the two ISC components $\mathrm{ISC}_{1}$ and $\mathrm{ISC}_{2}$ correspond to the two components $x_{1}(t)$ and $x_{2}(t)$.

Then, the wavelet parameters are optimized according to the signal itself using genetic algorithms. For $\mathrm{ISC}_{1}$, the optimal parameters are determined as $f_{b}=2.2316$ and $f_{c}=1.0532$. Figure 4 shows the evolution curve of GA. For ISC $_{2}$, the optimal parameters are $f_{b}=4.1203$ and $f_{c}=$ 1.0012. Ultimately, the signal is transformed self-adaptively using Morlet wavelet to extract wavelet ridge, and ISC $\mathrm{C}_{1}$ and $\mathrm{ISC}_{2}$ are demodulated based on the formula (10) and (11). The demodulation results are shown in Figures 5 and 6, respectively.

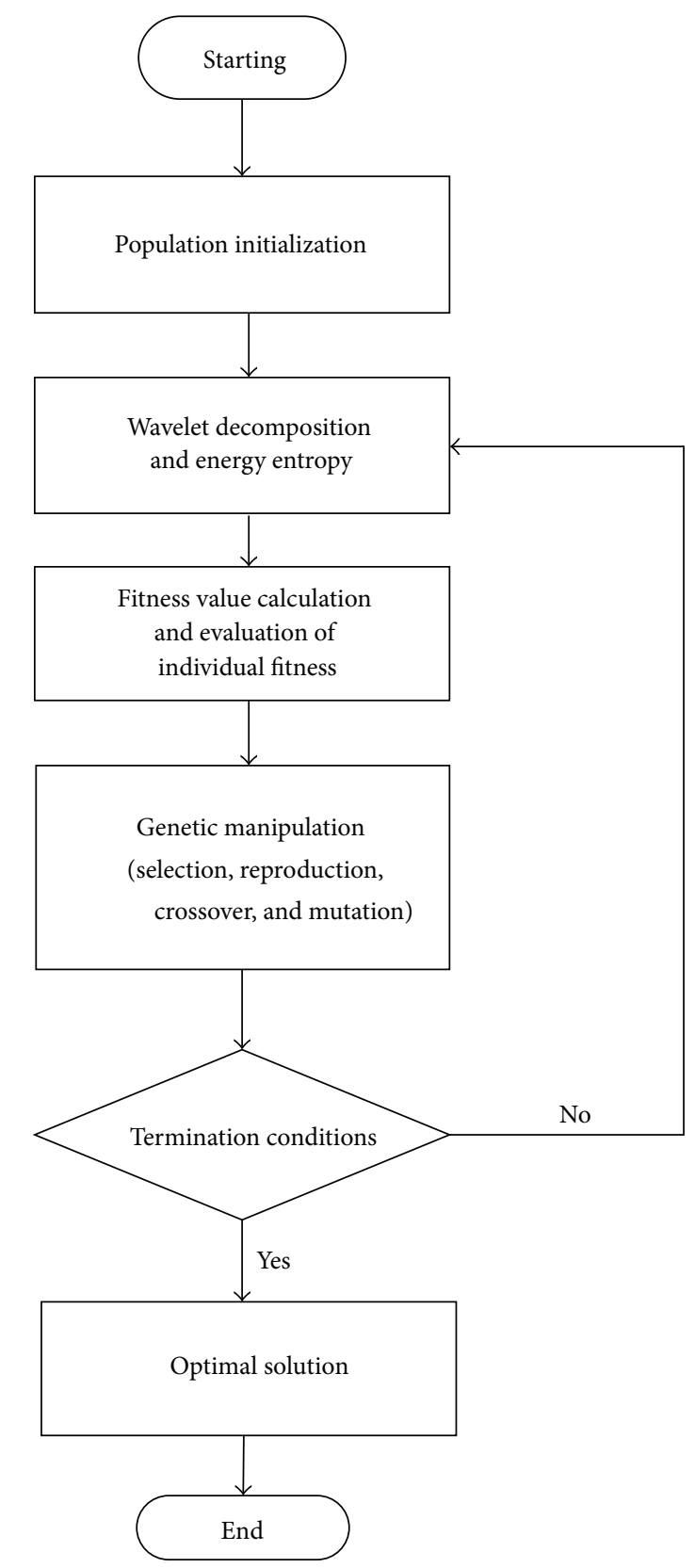

FIGURE 1: Flowchart of wavelet parameters optimization by GA.

For the instantaneous amplitude of ISC $_{1}$, to eliminate the border effect of the wavelet transformation, the boundary is processed by symmetric extension method. The result is given in Figure 7.

From the above analysis results, it can be seen that selfadaptive wavelet ridge demodulation method based on LCD can precisely demodulate a complex multicomponent AMFM signal. In order to make comparative analysis, the demodulation analysis results of $\mathrm{ISC}_{1}$ using Hilbert demodulation method are given in Figure 8, from which it can be found that the demodulation curve is not smooth and the demodulation error is bigger due to inevitable window effects by Hilbert demodulation method [4]. The above comparison results 


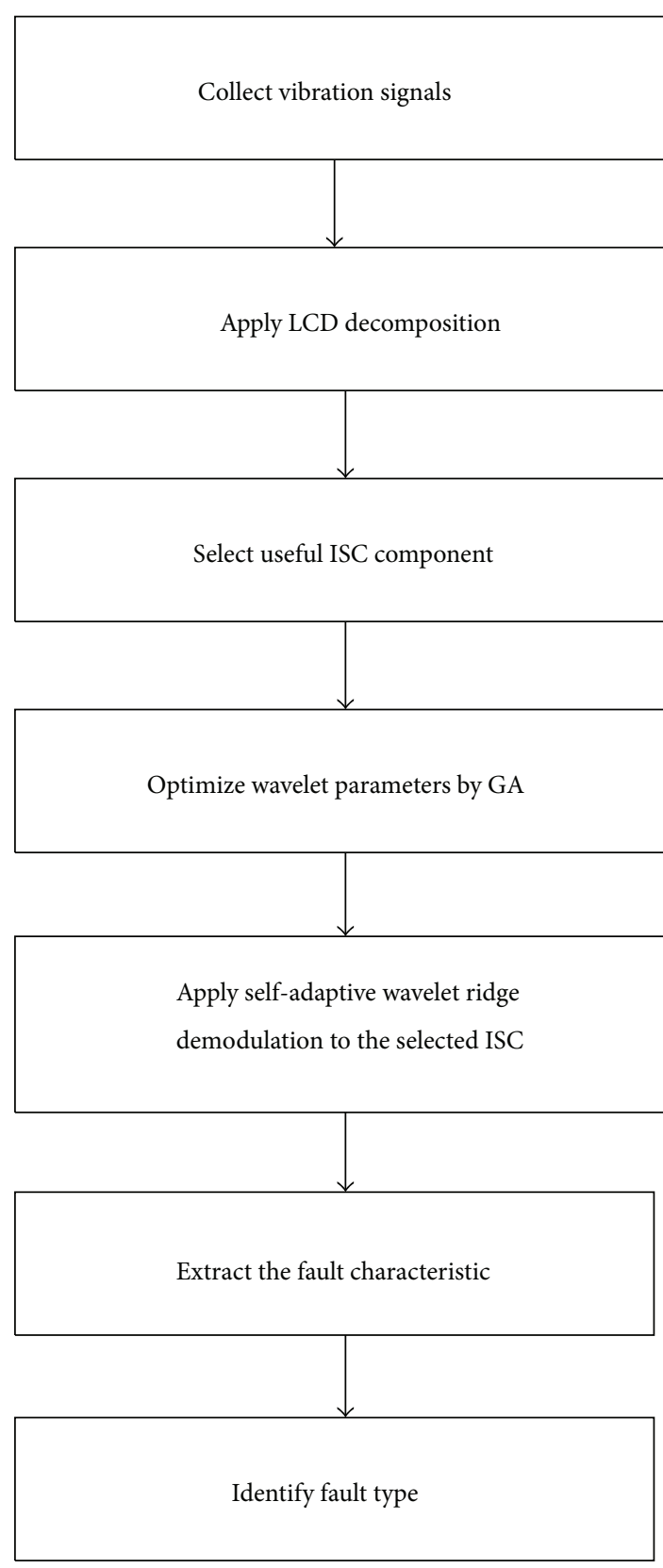

FIGURE 2: Flowchart of the proposed method.

indicate the proposed method is superior to the Hilbert demodulation method.

Let us consider another multicomponent AM-FM signal $y(t)$ which is a simulated faulty signal of gearbox and defined as follows:

$$
\begin{aligned}
& y(t)=y_{1}(t)+y_{2}(t), \\
& y_{1}(t)=[0.4+0.2 \cos (2 \pi \times 10 t)] \\
& \cdot \sin [2 \pi \times 600 t+\cos (2 \pi \times 20 t)], \\
& y_{2}(t)=[1+0.5 \cos (2 \pi \times 20 t)] \\
& \cdot \sin [2 \pi \times 300 t+\cos (2 \pi \times 15 t)] .
\end{aligned}
$$
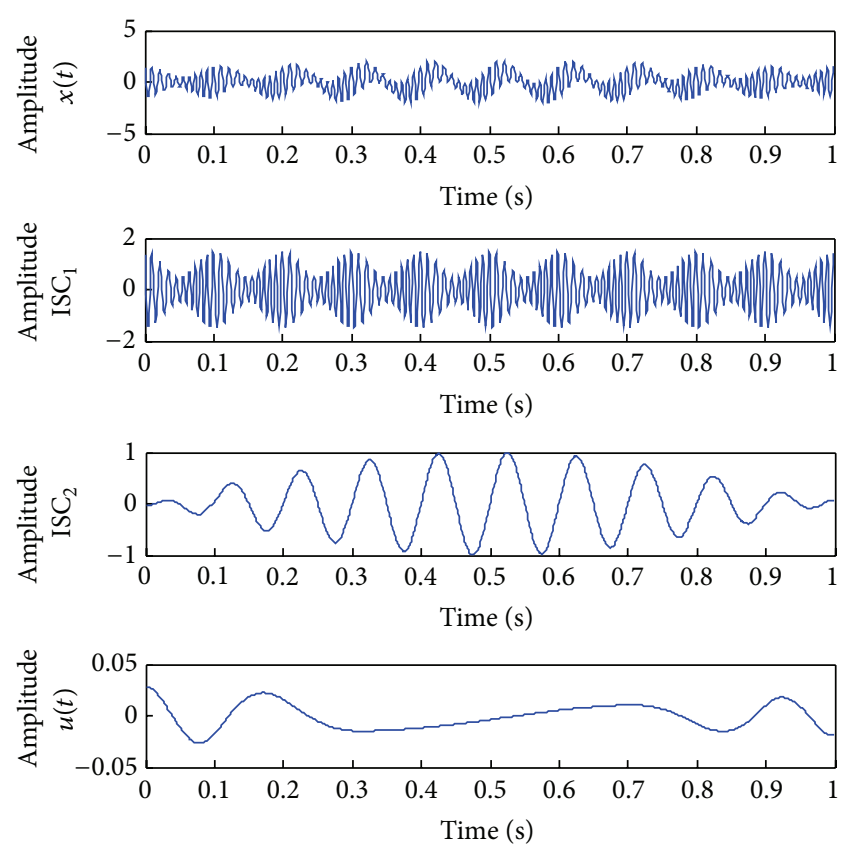

FIGURE 3: Time domain waveform of simulation signal $x(t)$ and its LCD decomposition result.

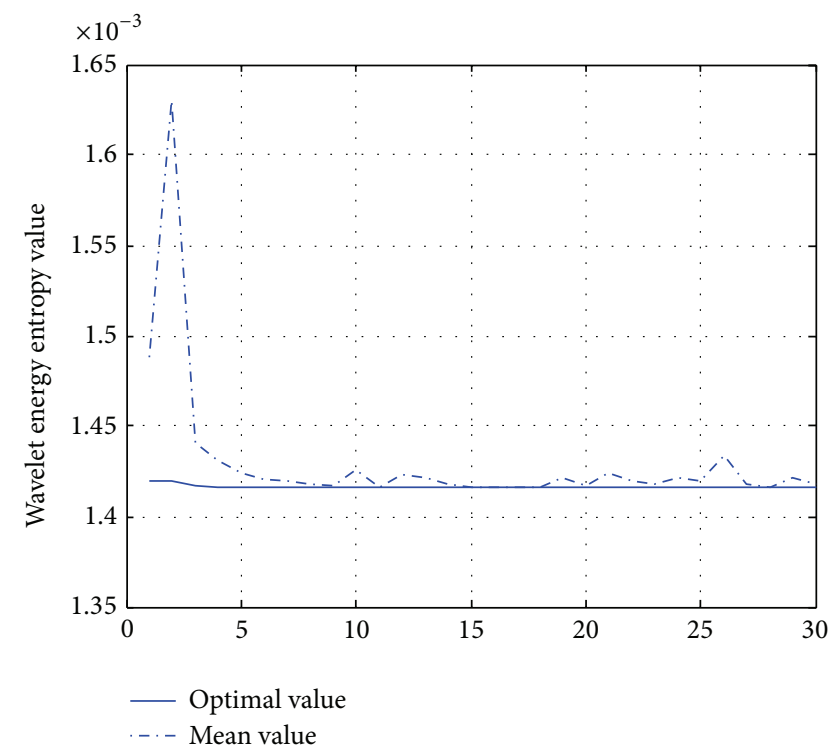

FIGURE 4: Evolution curve of GA.

The time domain waveform is shown in Figure 9. The sampling frequency is $2000 \mathrm{~Hz}$. Through LCD method, two ISC components are presented in Figure 10. Obviously, the two ISC components are consistent with the two components $y_{1}(t)$ and $y_{2}(t)$. By optimizing wavelet energy entropy, selfadaptive wavelet parameters for the first ISC signal are determined as $f_{b}=9.7752$ and $f_{c}=0.7428$, the parameters for the second ISC signal as $f_{b}=4.0585$ and $f_{c}=$ 1.0678. The self-adaptive wavelet transform results for the two ISCs are provided in Figures 11 and 12. It can be found that the IA's frequency of the first ISC signal is $10 \mathrm{~Hz}$ and 

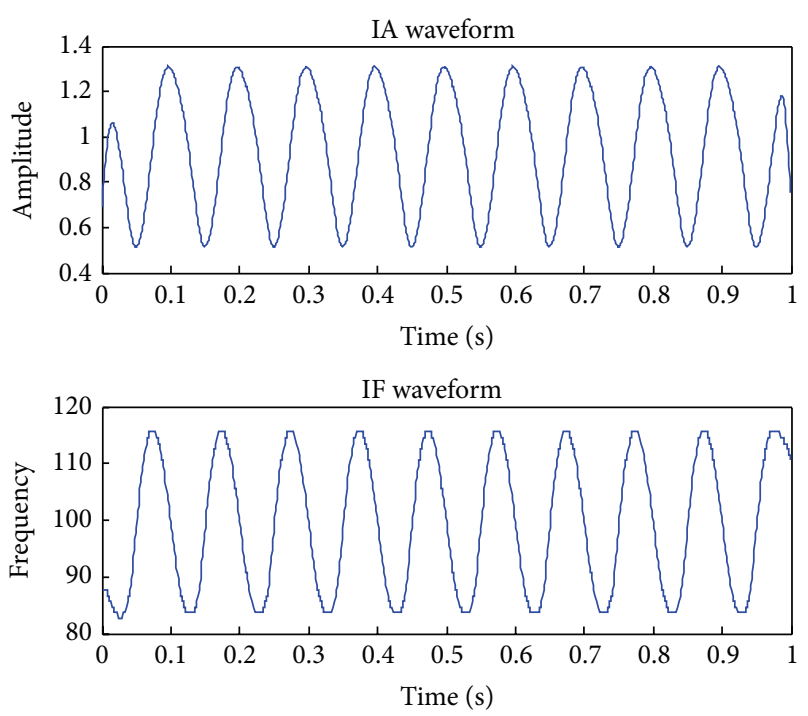

FIGURE 5: Self-adaptive wavelet ridge demodulation results of the 1st ISC.
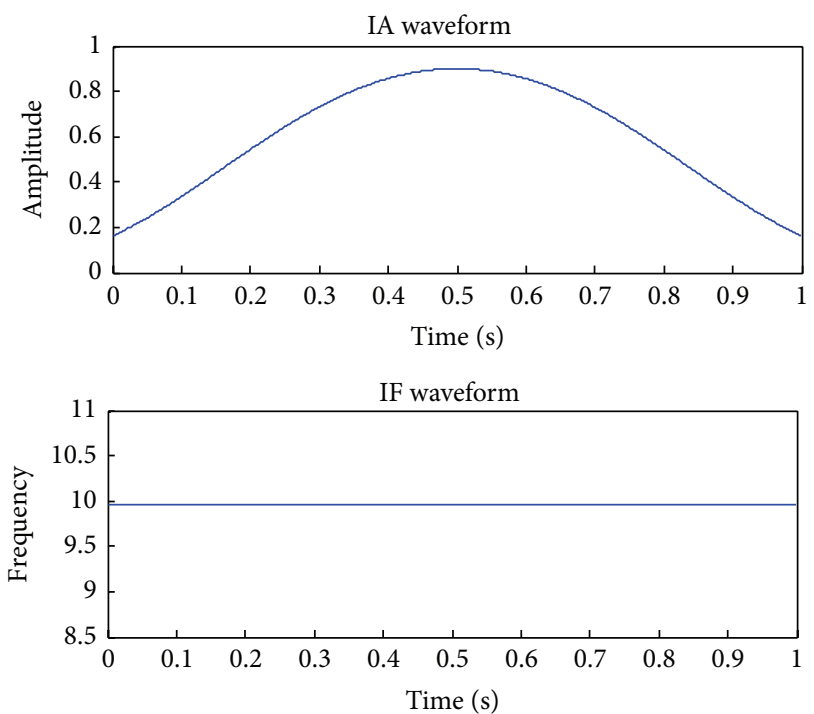

FIGURE 6: Self-adaptive wavelet ridge demodulation results of the 2nd ISC of simulation signal.

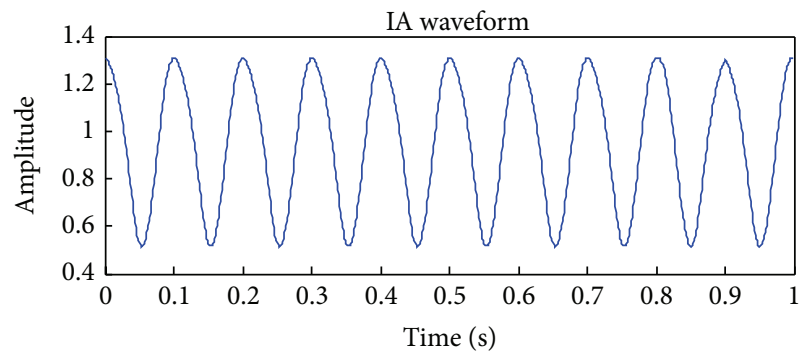

FIGURE 7: Processed instantaneous amplitude of the 1st ISC.
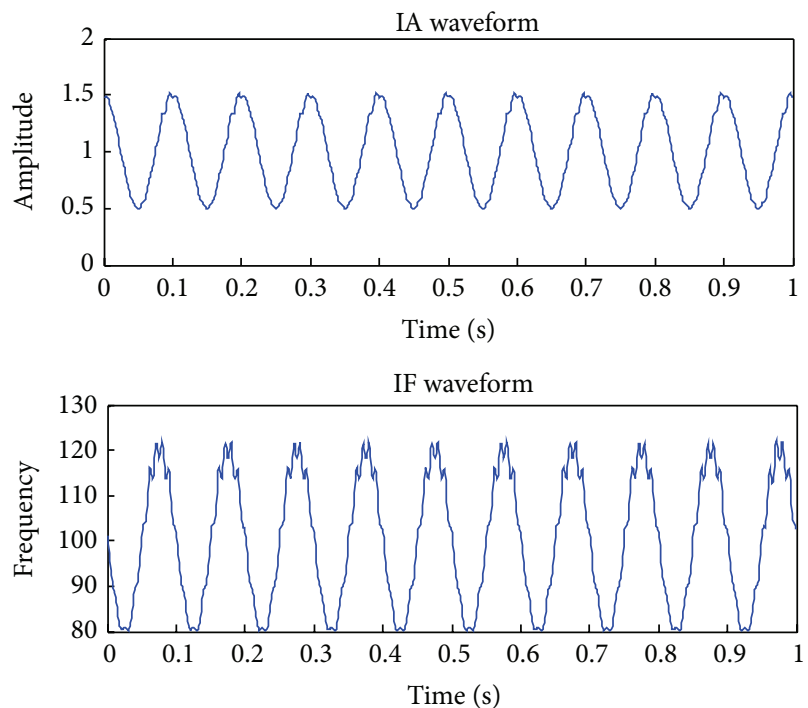

FIGURE 8: Hilbert demodulation results of the 1st ISC of simulation signal.

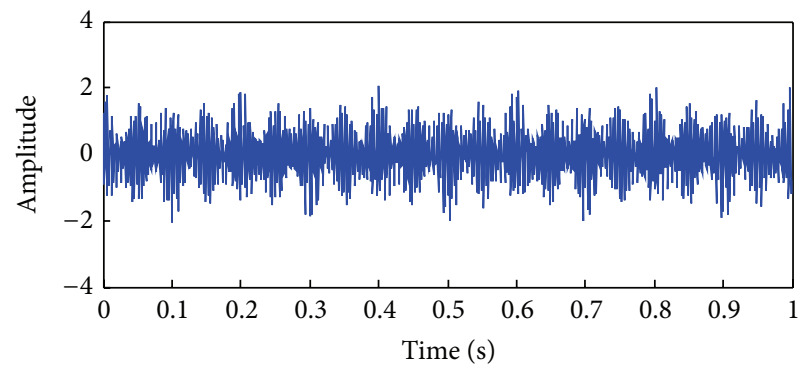

Figure 9: Waveform of simulation signal $y(t)$.
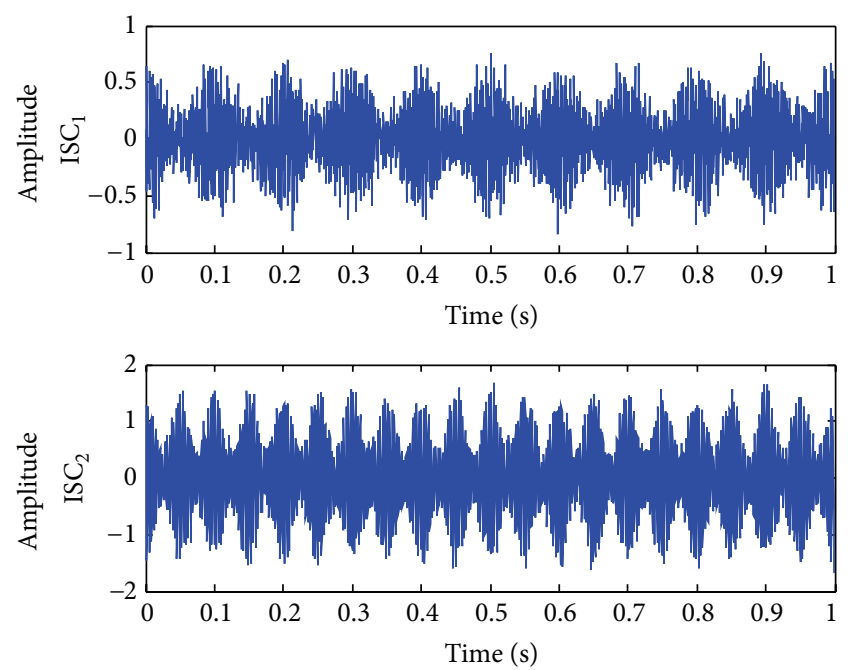

FIgURE 10: LCD results of simulation signal $y(t)$. 

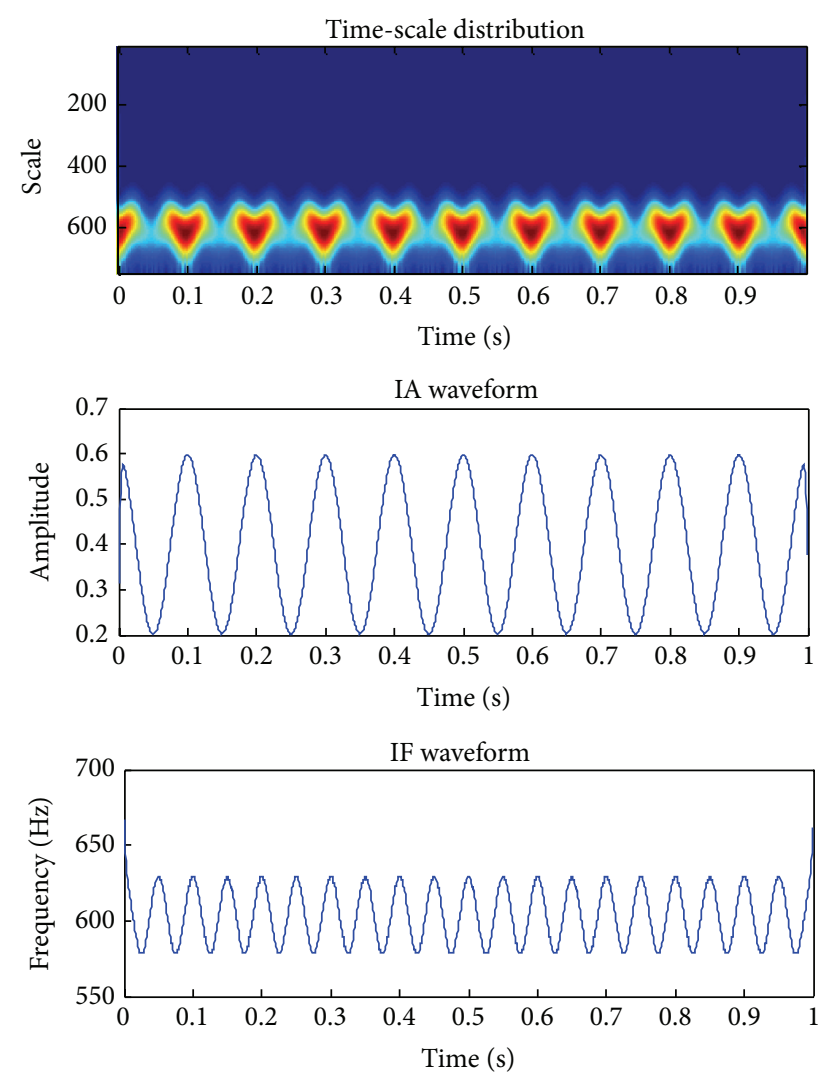

FIGURE 11: Demodulation results of the 1st ISC of simulation signal $y(t)$ by the proposed method.

the IF's frequency is $20 \mathrm{~Hz}$ and that IA's frequency of the second ISC signal is $20 \mathrm{~Hz}$ and the IF's frequency is $15 \mathrm{~Hz}$. These results are consistent with the two components in the original simulation signal. Therefore the proposed method can effectively demodulate multicomponent AM-FM signal and is suitable for fault diagnosis of gearbox.

For comparison, we use EMD method to make signal decomposition and use self-adaptive wavelet ridge demodulation approach to demodulate. We obtained eight IMFs in total. After analysis, we noted the first two IMFs reflected the modulation characteristics; hence they are considered as the two components. The demodulation results of the first two IMFs by self-adaptive wavelet ridge demodulation approach are provided in Figures 13 and 14. It can be seen that the FM component of the first IMF failed to be demodulated and the energy of the spectra of the second IMFs is lower. Then, energy operator demodulation based on EMD [4] is used and the results are shown in Figure 15. From these figures, it is shown that the serious mode mixing exists in the EMD decomposition, which influences the demodulation accuracy. However, from Figures 11 and 12, it is noted that LCD approach may diminish this problem and be superior to EMD approach.

Finally, we add a Gaussian white noise with deviation of 0.2 to $y(t)$. LCD decomposes the noisy signal into five ISCs. By the method introduced in Section 3.2, the optimal Morlet
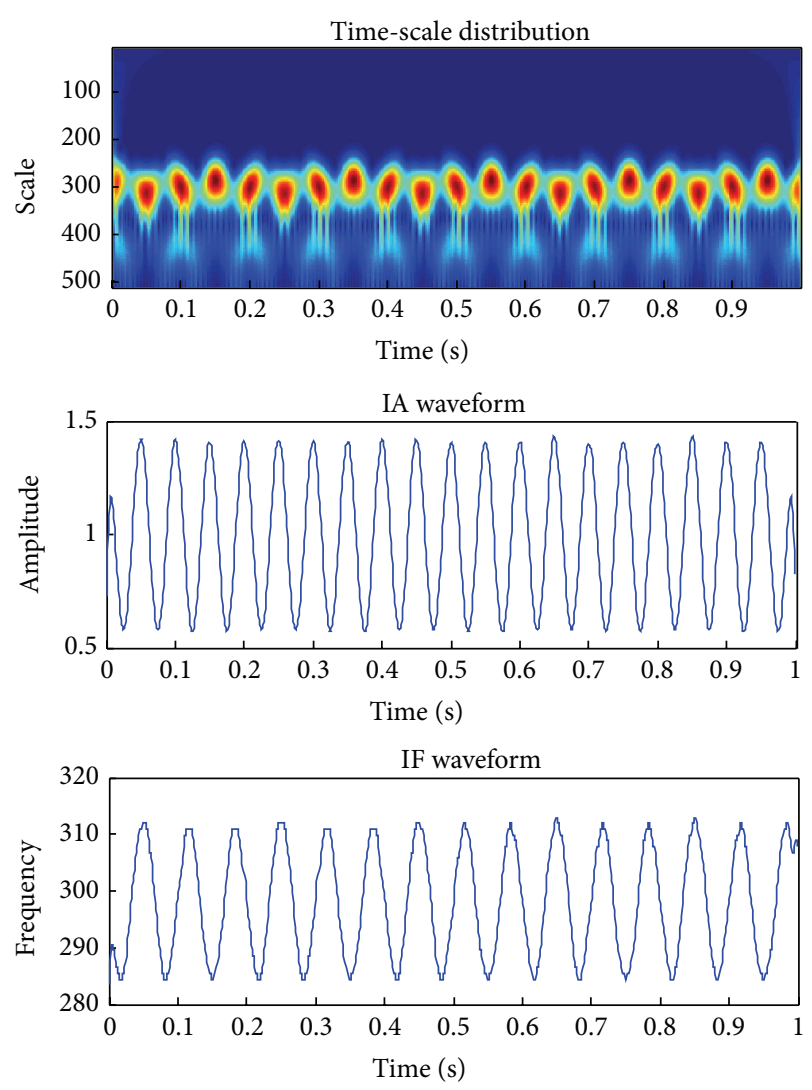

FIGURE 12: Demodulation results of the 2nd ISC of simulation signal $y(t)$ by the proposed method.

wavelet parameters for the first two ISCs, which contain modulation information, are determined. The demodulation results of the first two ISCs are shown in Figure 16. The results by energy operator demodulation based on LCD to the first two ISCs are given in Figure 17. It can be obviously found that self-adaptive wavelet demodulation approach based on LCD has better noise tolerant performance than energy operator demodulation approach based on LCD.

\section{Application to Incipient Fault Diagnosis}

5.1. Gear Crack Fault Diagnosis. A gear crack fault diagnosis experiment is carried out on bearing-gear test rig as shown in Figure 18. In this test, the motor power is $600 \mathrm{~W}$; both driving gear and driven gear are standard spur gear whose modulus is $2.5 \mathrm{~mm}$ and the number of teeth is 37 . The input and output shafts are arranged in parallel. They are supported by two roller bearings. A crack with $0.15 \mathrm{~mm}$ width and $1 \mathrm{~mm}$ depth at the root of the driving gear tooth is set by wire cutting machining to simulate the gear incipient crack failure. The vibration signals were collected by an accelerometer attached to the bearing housing. The shaft speed is $360 \mathrm{rev} / \mathrm{min}$; that is, the drive shaft rotation frequency is $f_{r}=6 \mathrm{~Hz}$. The sampling frequency is $1024 \mathrm{~Hz}$ and the length of sampling data is 1024 point. The domain waveform of the vibration signal measured 

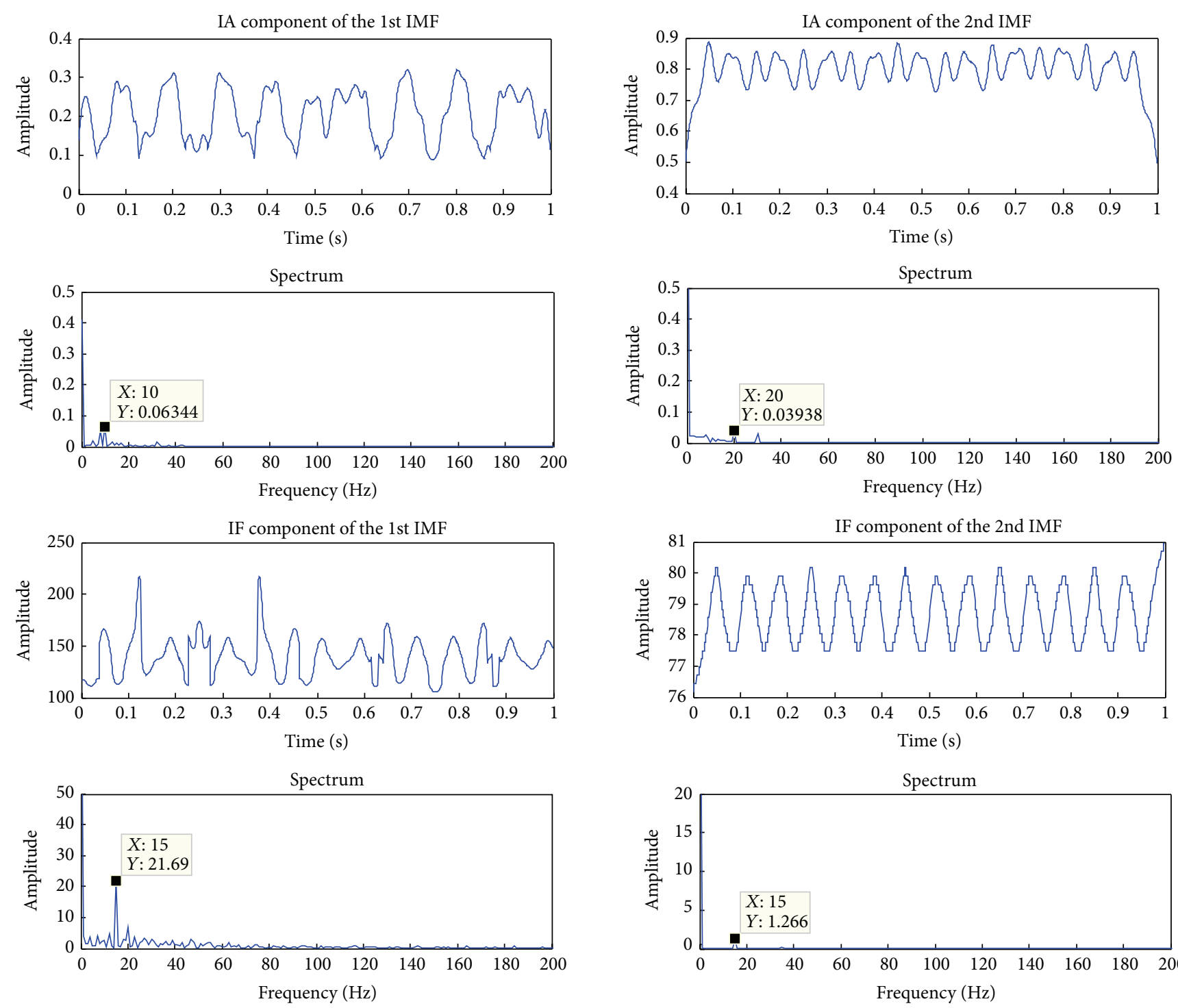

FIGURE 13: Demodulation results of the 1st IMF of simulation signal $y(t)$ by self-adaptive wavelet ridge demodulation approach based on EMD.

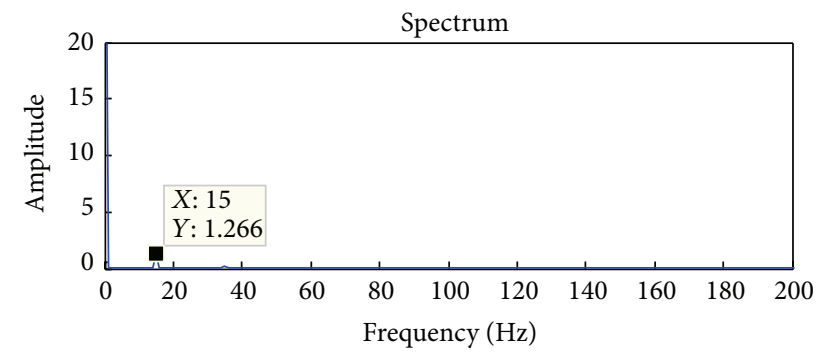

FIGURE 14: Demodulation results of the 2nd IMF of simulation signal $y(t)$ by self-adaptive wavelet ridge demodulation approach based on EMD.

and envelope spectrum are shown in Figure 19. From which it can be seen that fault characteristics are submerged by the background noise and the characteristics frequency fail to be identified.

Since the vibration signal with gear crack is a multicomponent AM-FM signal (AM-FM), we used the proposed method for fault diagnosis. Firstly, the vibration acceleration signal was decomposed into four ISC components $\mathrm{ISC}_{1} \sim \mathrm{ISC}_{4}$ and a residual component $r$ by LCD method, as shown in Figure 20. Because the carrier frequency of gear vibration signals is generally gear meshing frequency and its harmonics, we select the first ISC with the highest frequency for analysis. Figure 21 shows the Hilbert demodulation results of the first ISC, showing instantaneous amplitude contains complex high-frequency interference and some negative frequency

exists in the instantaneous frequency waveform, which is related to the measured signal.

Then, the optimum wavelet parameters $f_{b}=4.8574$ and $f_{c}=0.7832$ were selected. Lastly, the self-adaptive wavelet ridge demodulation was carried out. The IA waveform and its frequency spectrum obtained are shown in Figure 22, from which the fault characteristics frequency $f_{r}=6 \mathrm{~Hz}$ and its harmonics $3 f_{r}, 4 f_{r}$, and $5 f_{r}$ are clearly found. These demonstrate that a local defect has occurred in the gear on the drive shaft, which is consistent with the drive gear state. That is, the proposed method is effective for gear crack fault diagnosis.

In addition, we use the self-adaptive wavelet ridge demodulation approach to analyse the original signal as exhibited in Figure 23, where the spectrum contains the fault characteristics frequency and its harmonic as well, which 

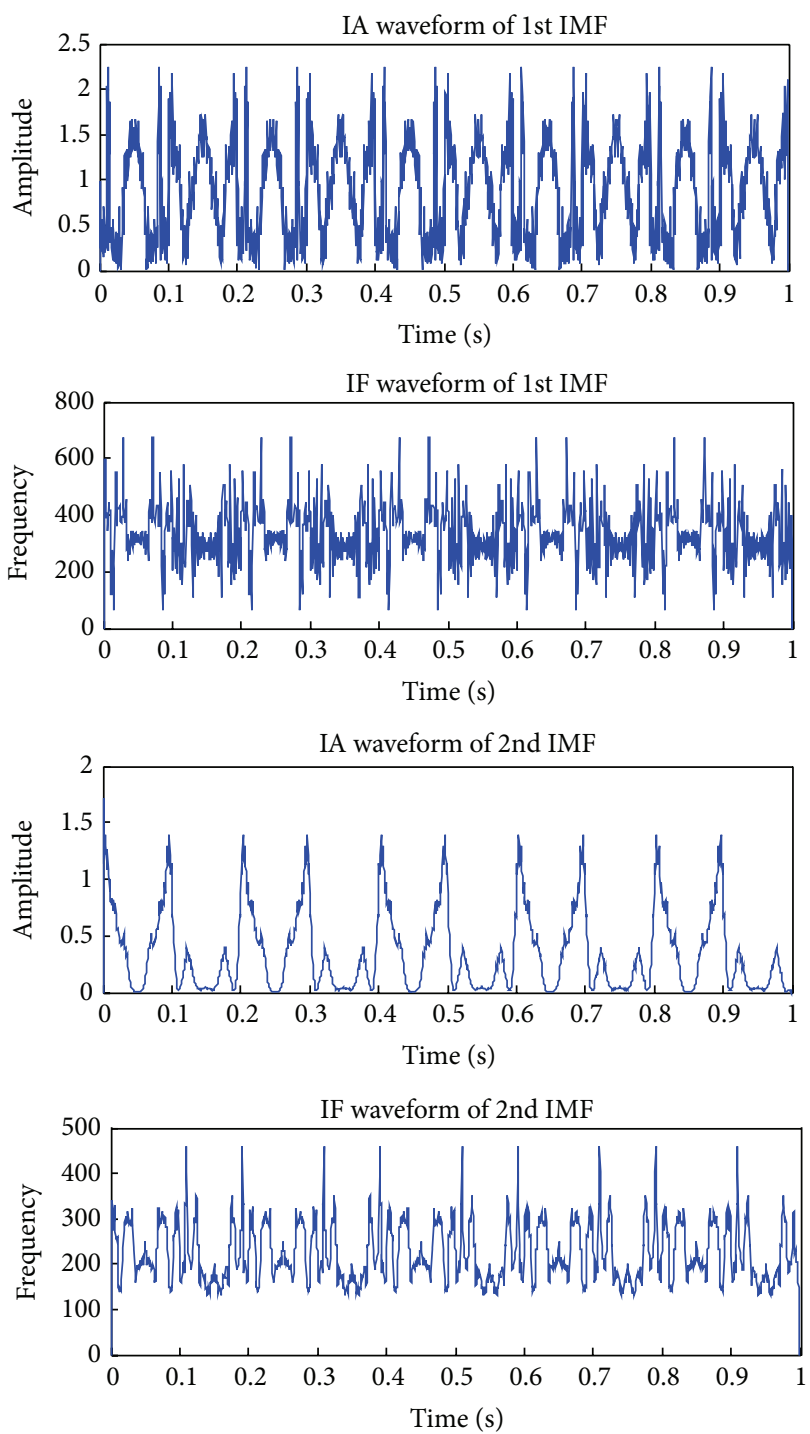

FIGURE 15: Demodulation results of simulation signal $y(t)$ by energy operator demodulation based on EMD.

outperform the excellent time-frequency localization ability of self-adaptive wavelet. But, some unknown frequency is involved because of background noise. Moreover, it can be noted that the harmonic in Figure 22 is richer and clearer than that in Figure 23. In fact, through signal decomposition by LCD and ISC selection, most of noise can be removed from analysis signal. Therefore, the proposed method is effective and superior in application to weak fault diagnosis for gear.

5.2. Bearing Inner-Race Fault Diagnosis. The vibration signal of roller bearing with inner-race fault is complex and weak; it is difficult to identify the fault state. To further verify the effectiveness of the proposed method, we made bearing inner-race fault diagnosis experiment.

The data was downloaded from the website of the Case Western Reserve University Bearing Center [24]. The test stand consists of a $2 \mathrm{hp}$ motor, a torque transducer, a dynamometer, and control electronics. The test bearing
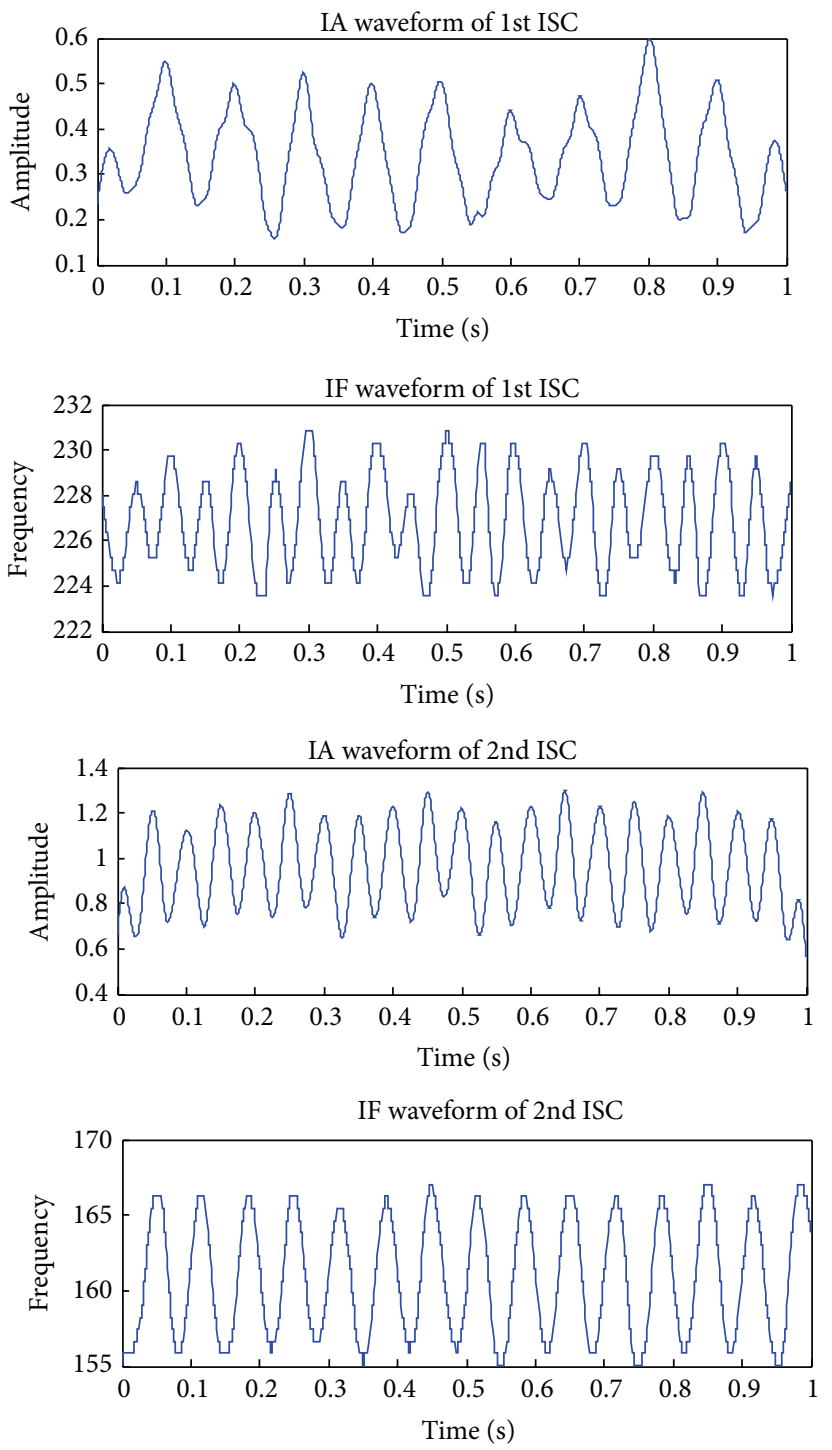

FIGURE 16: Demodulation results of simulation signal with noise by the proposed method.

is at the drive end; a single point defect was introduced into the inner raceway of the test bearing. The size of the single point defect is $0.178 \mathrm{~mm}$ in diameter and $0.279 \mathrm{~mm}$ in depth using electrodischarge machining. An accelerometer attached to the bearing housing collected vibration data with the sampling frequency as $12 \mathrm{kHz}$. The shaft rotating speed of the bearing inner-race is $1750 \mathrm{rev} / \mathrm{min}$; the characteristic frequency of the roller bearing with inner-race fault is $f_{o i}=$ $158 \mathrm{~Hz}$

Figure 24 presents the time domain waveform and spectrum of a bearing vibration signal. From the spectrum, it can be seen that there are three center frequency bands and their side frequency bands, which show that main modulation characteristics exhibits in the high frequency band. However, the fault characteristic frequency is not clear in the spectrum. Here, we used the proposed method to demodulate. The bearing vibration signal was decomposed into thirteen ISCs 

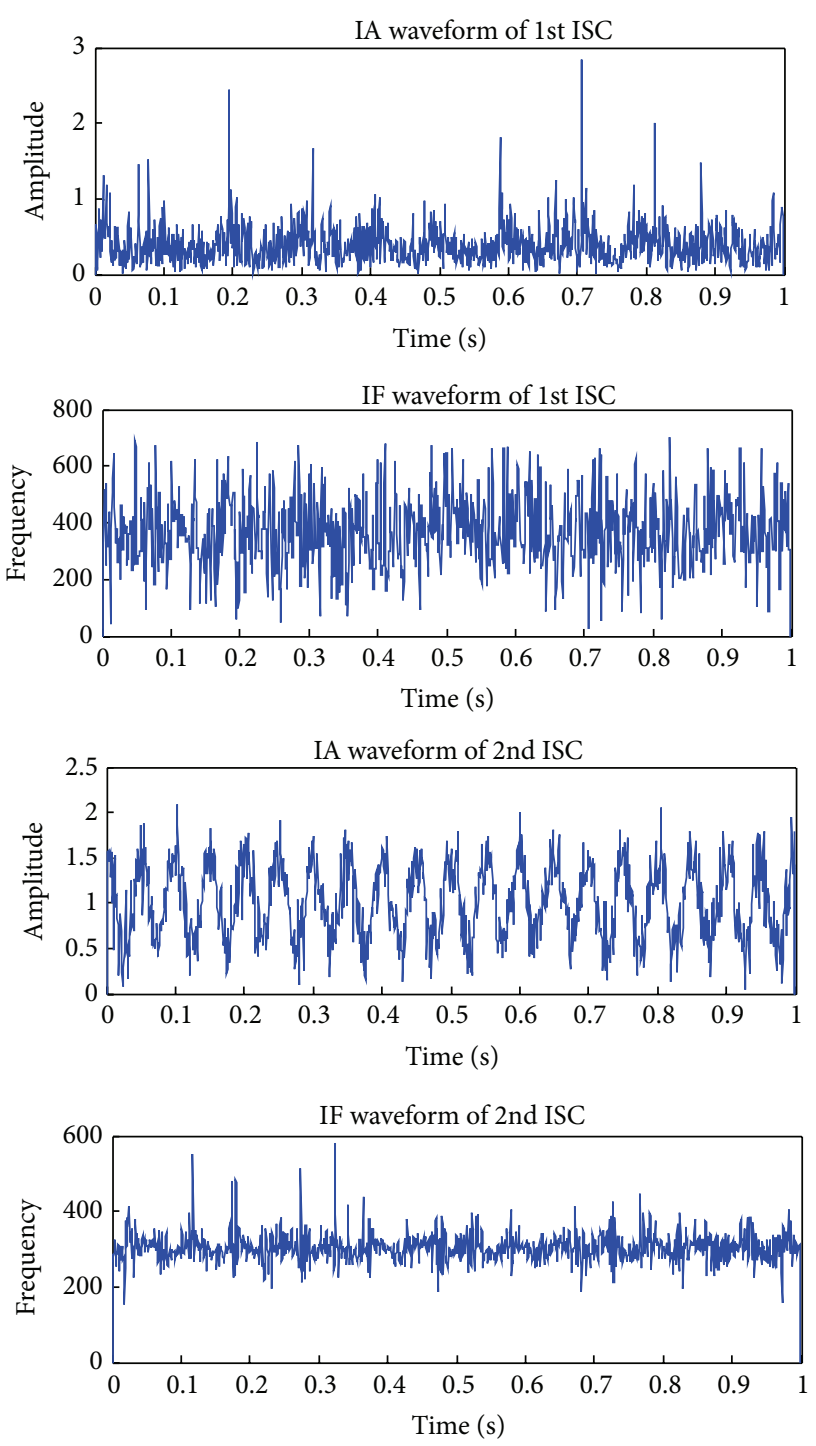

FIGURE 17: Demodulation results of simulation signal with noise by energy operator demodulation approach based on LCD.

and the first ISC with the highest frequency, as shown in Figure 25, was selected for analysis. The optimal Morlet wavelet parameters were determined as $f_{b}=12.8576$ and $f_{c}=0.6862$. The IA waveform and its spectrum are shown in Figure 26, from which we can clearly see the spectrum lines at the fault characteristic frequency $f_{o i}$ and its harmonics, which shows that a local failure occurs in the inner raceway of bearing. This is consistent with the fact state.

Besides, through comparison of Figure 24 with Figure 25, we can find that applying LCD decomposition equals to design adaptive band-pass filter, whose center frequency is automatically determined with the inherent characteristics of analyzed signal. Simultaneously, the selection of special ISC with higher frequency for analysis leads to decrease of the influence of lower frequency noise to suit weak fault feature extraction. In addition, due to concern of main

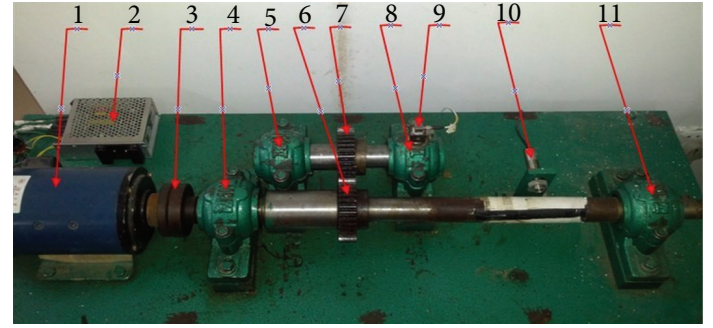

(1) Induction motor

(2) Speed controller

(3) Coupling

(4) Bearing number 1 and bearing housing

(5) Bearing number 2 and bearing housing

(6) Drive gear

(7) Driven gear

(8) Bearing number 3 and bearing housing

(9) Vibration acceleration sensor

(10) Speed sensor

(11) Bearing number 4 and bearing housing

FIGURE 18: Bearing-gear test rig for vibration signal acquisition.
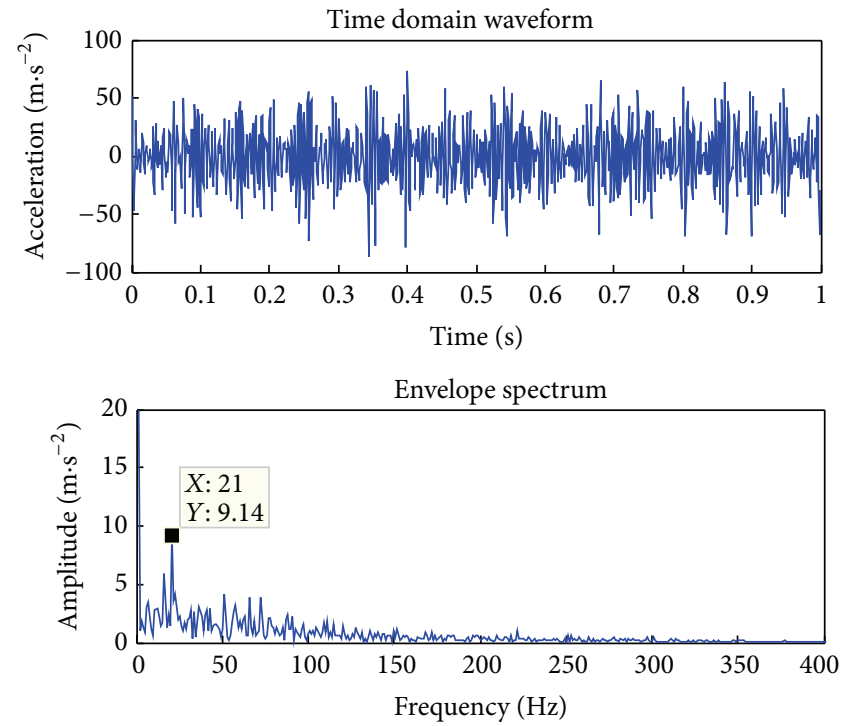

FIgURE 19: Time domain waveform and envelope spectrum of a faulty gear vibration signal.

energy on the time-scale distribution, self-adaptive wavelet ridge modulation has excellent time-frequency localization property, as seen in Figure 23. Therefore, the application results show the proposed method is simple and effective for weak bearing inner-race fault diagnosis.

\section{Conclusion}

LCD method is a new signal decomposition approach, which is suitable to preprocess the multicomponent AMFM signal. Wavelet ridge demodulation method is based on wavelet transform in the time-frequency domain, focusing 


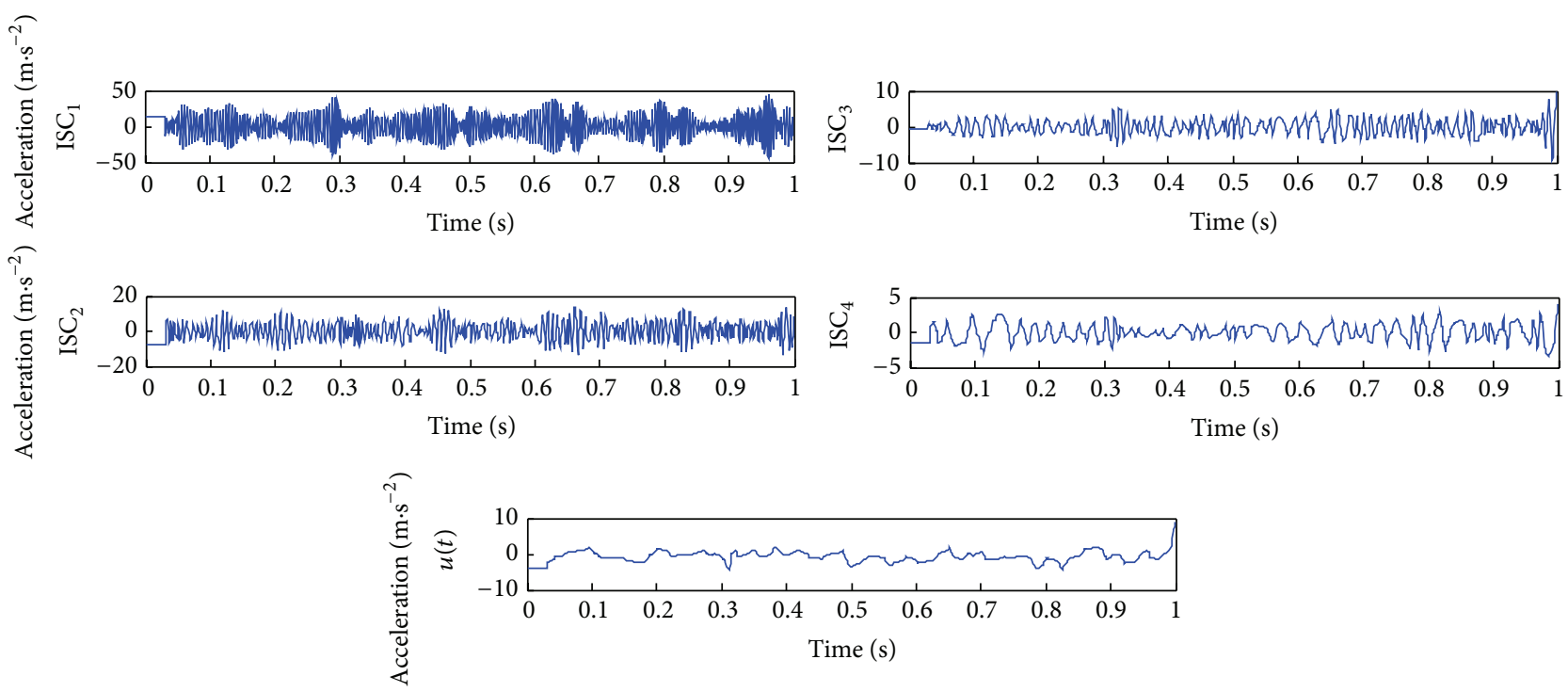

FIGURE 20: LCD decomposition results of a faulty gear vibration signal.
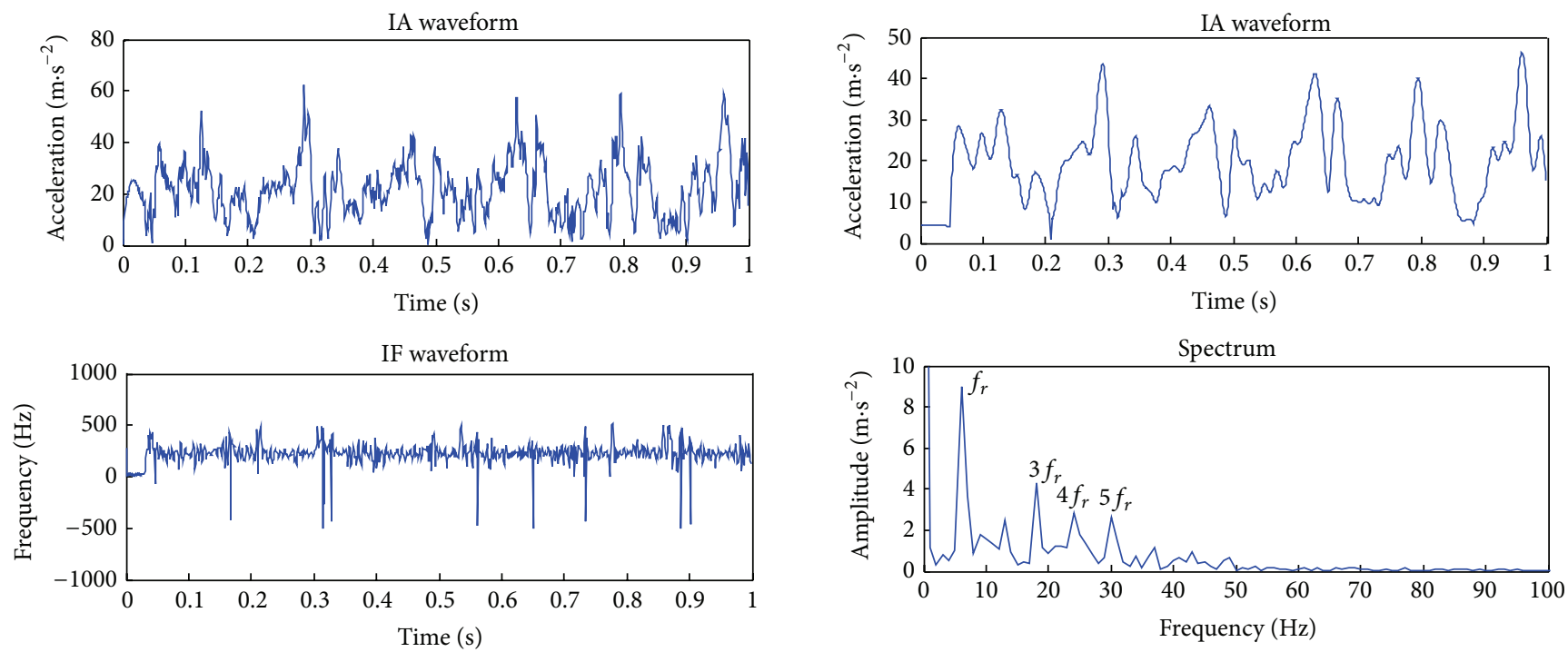

FIGURE 21: Demodulation results of the 1st ISC by Hilbert approach.

on main energy on the time-scale distribution. It is less sensitive to signal bandwidth and noise, especially using the wavelet transform with optimal parameters, called selfadaptive wavelet. Therefore, with combination of the above two approaches, a self-adaptive wavelet ridge demodulation method based on LCD for fault diagnosis is presented.

Using two simulation signals, we compare the proposed method with the following four approaches: Hilbert demodulation, energy operator demodulation based on EMD, selfadaptive wavelet ridge demodulation based on EMD, and energy operator demodulation based on LCD. Analysis
FIGURE 22: Instantaneous amplitude and its spectrum of the 1st ISC by the proposed method.

results show that the proposed method has higher demodulation accuracy and higher noise tolerant performance than the others. Finally, we applied the proposed method to incipient fault diagnosis of gearbox. The application results show the proposed method is simple and effective for incipient fault diagnosis of gearbox.

\section{Conflict of Interests}

The authors declare that there is no conflict of interests regarding the publication of this paper. 

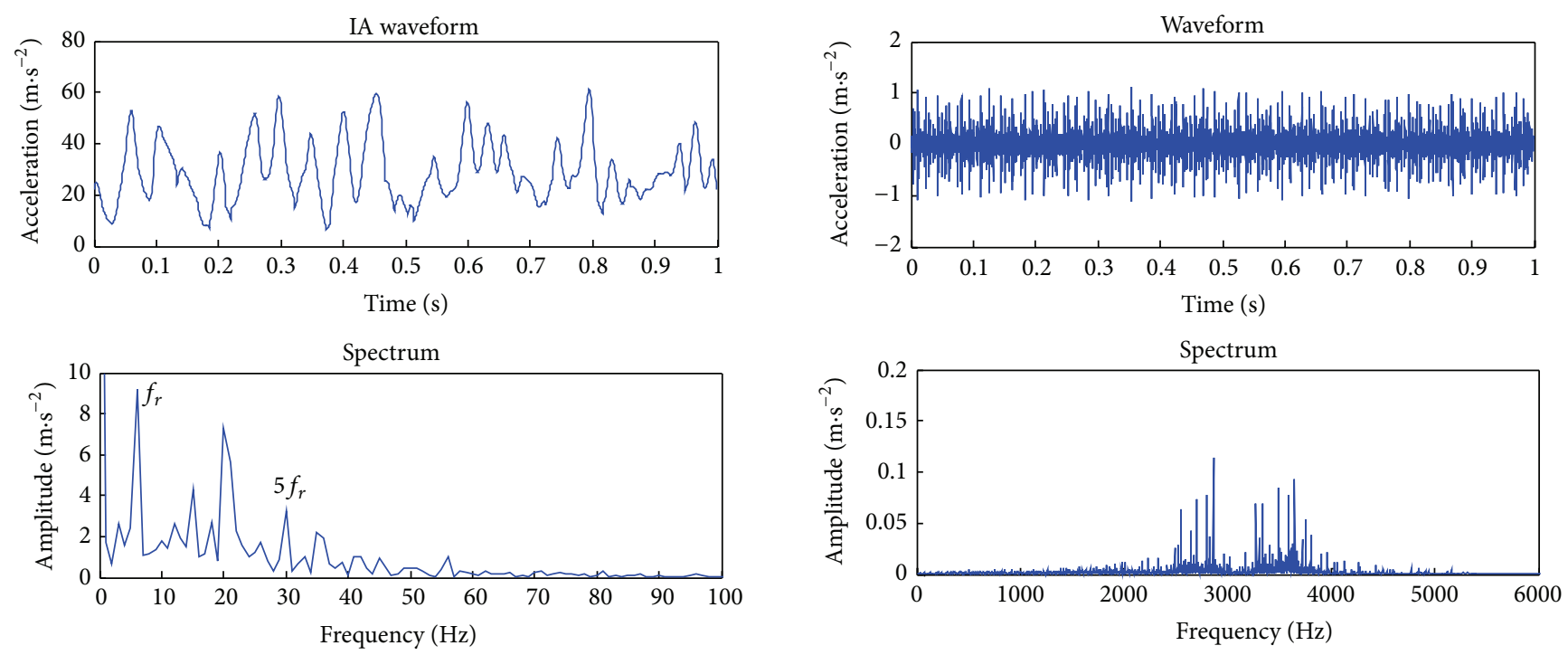

FIGURE 23: Instantaneous amplitude and its spectrum of the original vibration signal by self-adaptive wavelet ridge demodulation approach.
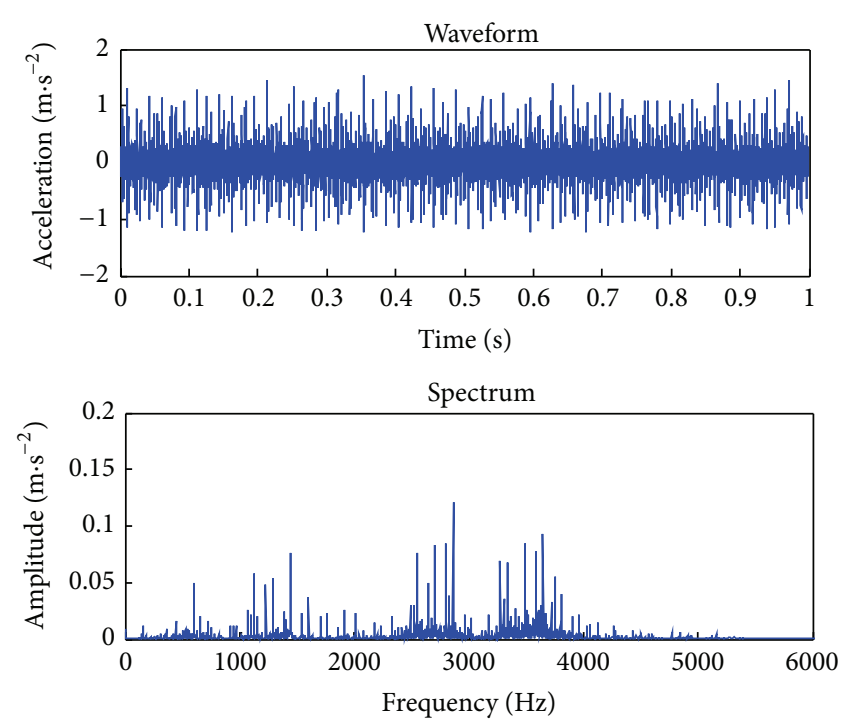

FIGURE 25: Time domain waveform and spectrum of the 1st ISC of a roller bearing vibration signal with inner-race defect.
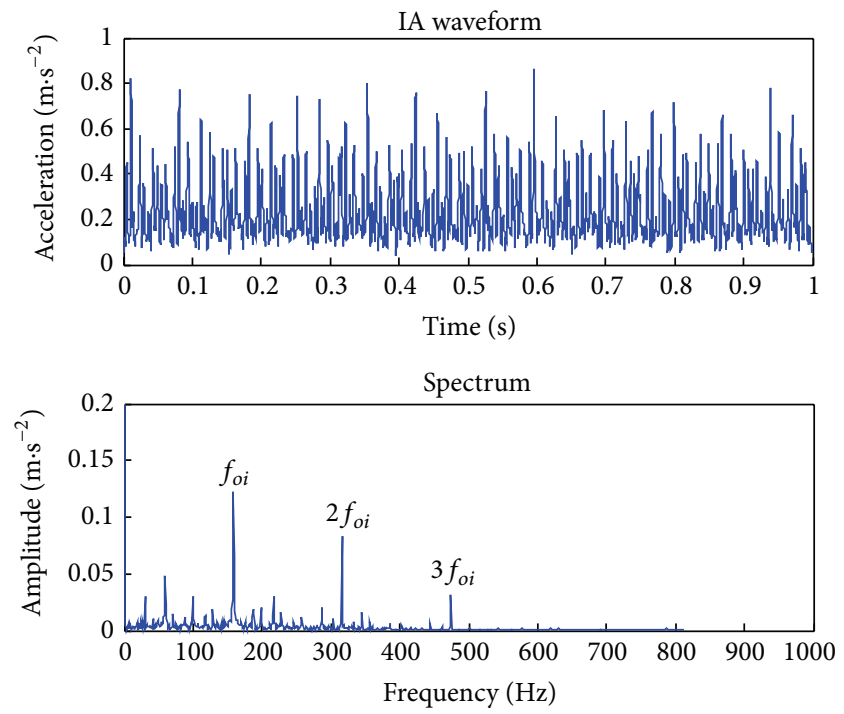

FIGURE 24: Time domain waveform and spectrum of a roller bearing vibration signal with inner-race defect.

\section{Acknowledgments}

The support from Chinese National Science Foundation Grant (no. 51075131), the construct program of the key discipline in Hunan Province (Mechanical Design and Theory) (XJF2011 [76]), cooperative Demonstration Base of Universities in Hunan " $\mathrm{R} \& \mathrm{D}$ and Industrialization of Rock Drilling Machines” (XJT [2014] 239), Hunan Major Special Projects of Science and Technology (2014GK1043), and scientific research project of Hunan Province Education Department (no. 14C0789) is greatly acknowledged. The authors would like to express their appreciation to Case Western Reserve University for offering the free bearing data.

FIGURE 26: Instantaneous amplitude waveform and spectrum of the 1st ISC of a roller bearing vibration signal with inner-race defect.

\section{References}

[1] R. B. Randall, "A new method of modeling gear faults," ASME Journal of Mechanical Design, vol. 104, no. 2, pp. 259-267, 1982.

[2] M. Feldman, "Nonparametric identification of asymmetric nonlinear vibration systems with the Hilbert transform," Journal of Sound and Vibration, vol. 331, no. 14, pp. 3386-3396, 2012.

[3] N. E. Huang, Z. Shen, S. R. Long et al., "The empirical mode decomposition and the Hilbert spectrum for nonlinear and non-stationary time series analysis," The Royal Society of London. Proceedings. Series A. Mathematical, Physical and Engineering Sciences, vol. 454, no. 1971, pp. 903-995, 1998.

[4] J. Cheng, D. Yu, and Y. Yang, "The application of energy operator demodulation approach based on EMD in machinery 
fault diagnosis," Mechanical Systems and Signal Processing, vol. 21, no. 2, pp. 668-677, 2007.

[5] Z. Feng, M. Liang, Y. Zhang, and S. Hou, "Fault diagnosis for wind turbine planetary gearboxes via demodulation analysis based on ensemble empirical mode decomposition and energy separation," Renewable Energy, vol. 47, pp. 112-126, 2012.

[6] Y. Qin, "Multicomponent AM-FM demodulation based on energy separation and adaptive filtering," Mechanical Systems and Signal Processing, vol. 38, no. 2, pp. 440-459, 2013.

[7] M. Liang and H. Faghidi, "Intelligent bearing fault detection by enhanced energy operator," Expert Systems with Applications, vol. 41, no. 16, pp. 7223-7234, 2014.

[8] J. Wang and Q. He, "Exchanged ridge demodulation of timescale manifold for enhanced fault diagnosis of rotating machinery," Journal of Sound and Vibration, vol. 333, no. 11, pp. 24502464, 2014.

[9] Y. Qin, S. Qin, and Y. Mao, "Demodulation approach based on wavelet ridge and its application in fault diagnosis of rotating machinery," Chinese Journal of Mechanical Engineering, vol. 45, no. 2, pp. 231-237, 2009.

[10] W. He, Z. Jiang, and Q. Qin, "A joint adaptive wavelet filter and morphological signal processing method for weak mechanical impulse extraction," Journal of Mechanical Science and Technology, vol. 24, no. 8, pp. 1709-1716, 2010.

[11] Y. Jiang, B. Tang, Y. Qin, and W. Liu, "Feature extraction method of wind turbine based on adaptive Morlet wavelet and SVD," Renewable Energy, vol. 36, no. 8, pp. 2146-2153, 2011.

[12] W. Su, F. Wang, H. Zhu, Z. Zhang, and Z. Guo, "Rolling element bearing faults diagnosis based on optimal Morlet wavelet filter and autocorrelation enhancement," Mechanical Systems and Signal Processing, vol. 24, no. 5, pp. 1458-1472, 2010.

[13] H. Qiu, J. Lee, J. Lin, and G. Yu, "Wavelet filter-based weak signature detection method and its application on rolling element bearing prognostics," Journal of Sound and Vibration, vol. 289, no. 4-5, pp. 1066-1090, 2006.

[14] M. Amarnath and I. Praveen Krishna, "Local fault detection in helical gears via vibration and acoustic signals using EMD based statistical parameter analysis," Measurement, vol. 58, pp. 154$164,2014$.

[15] Y. Lei, J. Lin, Z. He, and M. J. Zuo, "A review on empirical mode decomposition in fault diagnosis of rotating machinery," Mechanical Systems and Signal Processing, vol. 35, no. 1-2, pp. 108-126, 2013.

[16] J. S. Smith, "The local mean decomposition and its application to EEG perception data," Journal of the Royal Society Interface, vol. 2, no. 5, pp. 443-454, 2005.

[17] H. Liu and M. Han, "A fault diagnosis method based on local mean decomposition and multi-scale entropy for roller bearings," Mechanism and Machine Theory, vol. 75, pp. 67-78, 2014.

[18] J. Cheng and Y. Yang, "A rotating machinery fault diagnosis method based on local mean decomposition," Digital Signal Processing, vol. 22, no. 2, pp. 356-366, 2012.

[19] J. Zheng, J. Cheng, and Y. Yang, "A rolling bearing fault diagnosis approach based on LCD and fuzzy entropy," Mechanism and Machine Theory, vol. 70, pp. 441-453, 2013.

[20] J. Zheng, J. Cheng, Y. Nie, and S. Luo, "A signal processing method for fault diagnosis-complete ensemble local characteristic-scale decomposition," Journal of Vibration Engineering, vol. 27, no. 4, pp. 637-646, 2014.
[21] H. L. Ao, J. Cheng, K. Li, and T. K. Truong, "A roller bearing fault diagnosis method based on LCD energy entropy and ACROASVM," Shock and Vibration, vol. 2014, Article ID 825825, 12 pages, 2014.

[22] M. Unal, M. Onat, M. Demetgul, and H. Kucuk, "Fault diagnosis of rolling bearings using a genetic algorithm optimized neural network," Measurement, vol. 58, pp. 187-196, 2014.

[23] B. Long, W. Xian, M. Li, and H. Wang, "Improved diagnostics for the incipient faults in analog circuits using LSSVM based on PSO algorithm with Mahalanobis distance," Neurocomputing, vol. 133, no. 10, pp. 237-248, 2014.

[24] CWRU, Bearing Data Center, Seeded Fault Test Data, 2013, http://csegroups.case.edu/bearingdatacenter/home. 

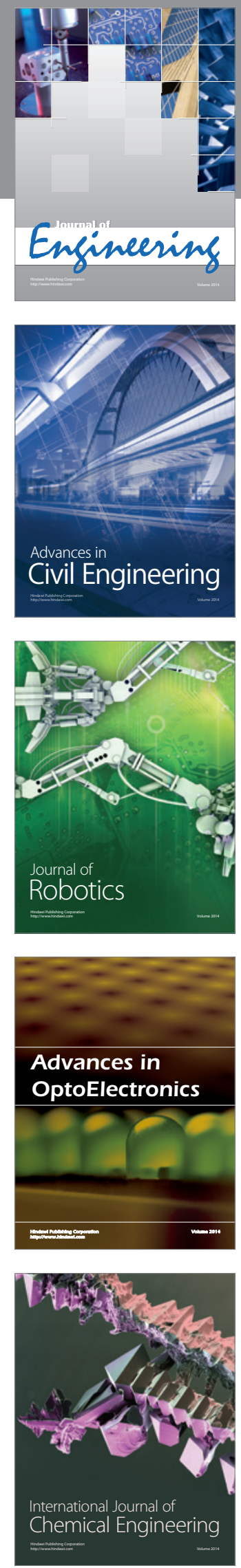

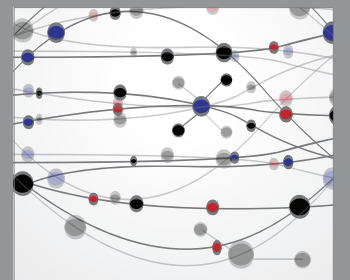

The Scientific World Journal
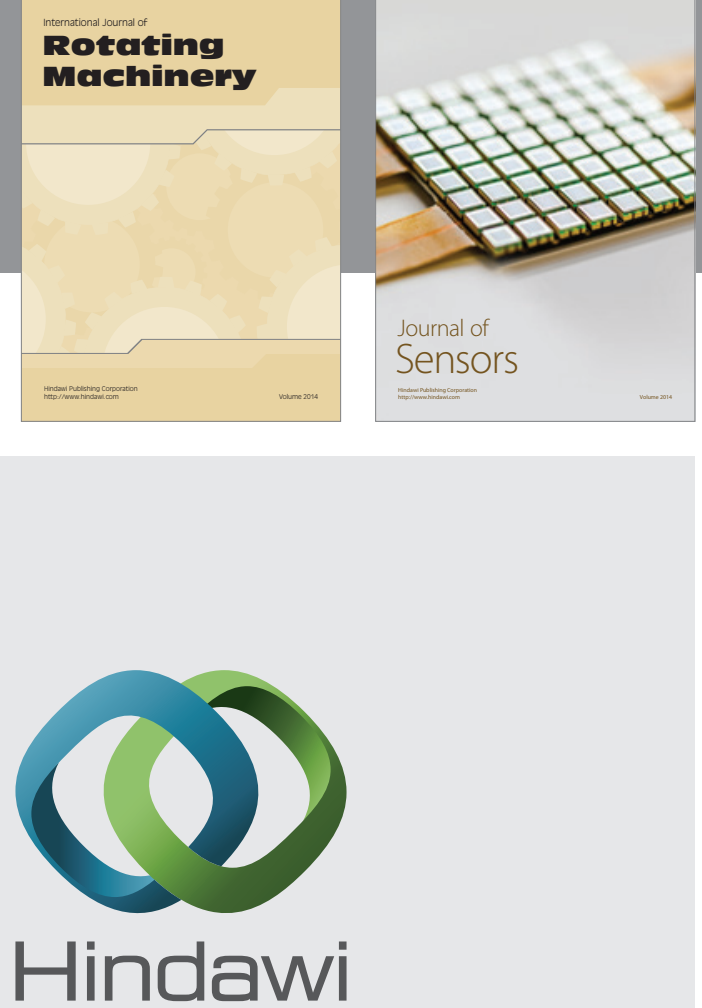

Submit your manuscripts at http://www.hindawi.com
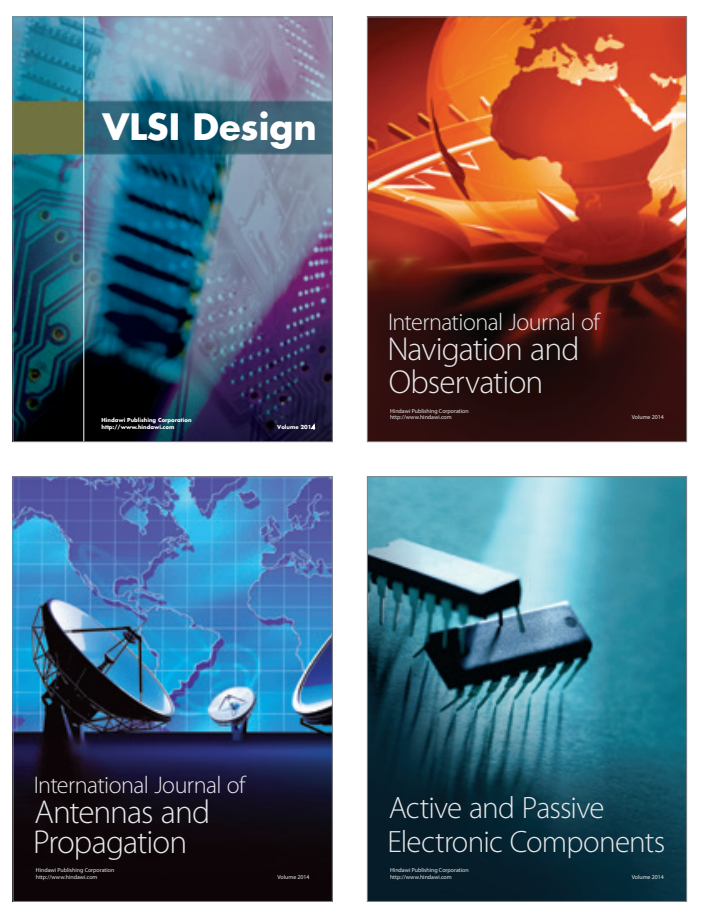
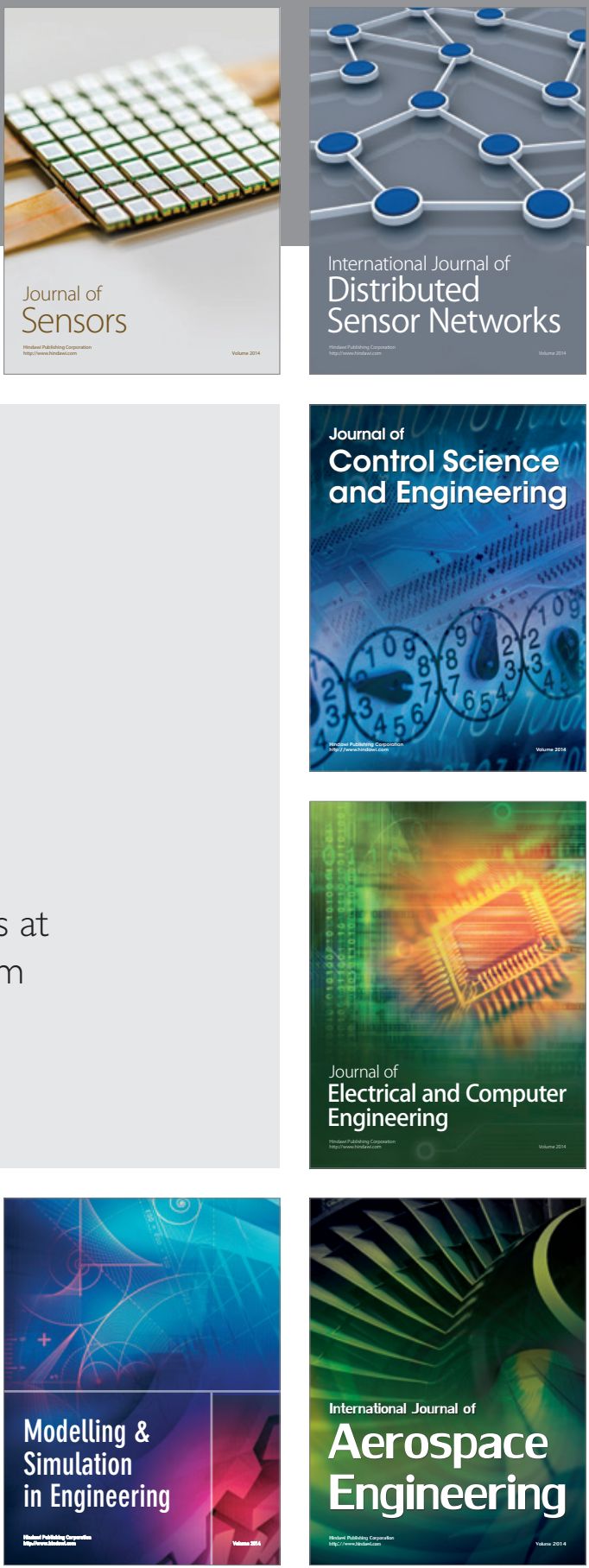

Journal of

Control Science

and Engineering
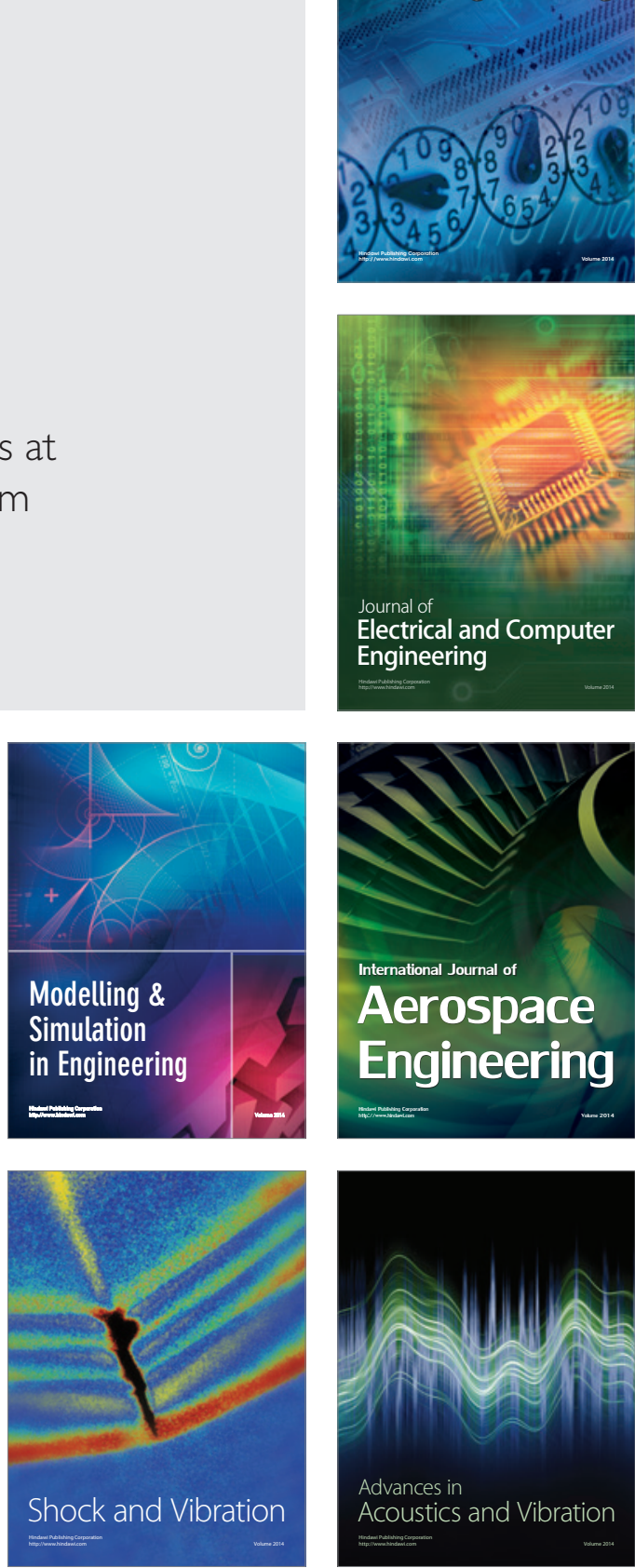